\title{
P-mSA/mCS 双极膜的制备及其在一价、二价离子分离中的应用
}

\author{
肖艳春陈日耀 郑曦陈晓陈震
}

(福建师范大学化学与材料学院, 福州 350007)

\begin{abstract}
摘要: 以五氧化二磷、磷酸三乙酯和磷酸为反应剂, 制备了磷酸化海藻酸钠 $(\mathrm{P}-\mathrm{SA})$, 经二茂铁离子改性后作为 阳膜层(P-mSA); 用乙酰基二茂铁改性壳聚糖制备了阴膜溶胶(mCS). 将阴膜溶胶流延于阳膜层上, 制备了 $\mathrm{P}-二$ 茂铁-SA/乙酰基二茂铁-CS 双极膜(P-mSA/mCS BPM). 测定了双极膜的红外光谱、接触角、电荷密度、离子交换 容量与交流阻抗. IR 与接触角的分析结果表明, SA 经磷酸化后, 亲水性能得到了显著提高. 将 P-mSA/mCS BPM 应用于一价、二价离子的分离, 当压力差为 $0.3 \mathrm{MPa}$ 时, 二价离子的截留率(双极膜截留的离子百分数)为 $95 \%$, 一价离子的截留率为 $22 \%$ 左右.
\end{abstract}

关键词：双极膜; 磷酸化; 二茂铁; 一价、二价离子的分离

中图分类号: 0646

\section{Preparation of P-mSA/mCS Bipolar Membrane and Its Application in Separating Monovalent and Divalent Ions}

\author{
XIAO Yan-Chun CHEN Ri-Yao ZHENG Xi CHEN Xiao CHEN Zhen* \\ (College of Chemistry and Materials Science, Fujian Normal University, Fuzhou 350007, P. R. China)
}

\begin{abstract}
A phosphorylated sodium alginate (P-SA) membrane was synthesized using phosphorus pentaoxide, riethyl phosphate and phosphoric acid and then modified by ferrocene. Chitosan (CS) was modified by acetylferrocene. A P-ferrocene-SA/acetylferrocene-CS bipolar membrane, P-mSA/mCS BPM, was prepared by a paste method. The bipolar membrane was characterized by Fourier transform infrared spectroscopy (FTIR), contact angle, charge density, ion exchange capacity and alternating current impedance spectroscopy. Results of FTIR and contact angle measurements showed that the hydrophilic property of SA improved after modification by phosphorylation. P-mSA/ mCS BPM was applied to separate monovalent and divalent ions. The rejection ratios of divalent and monovalent ions were about $95 \%$ and $22 \%$, respectively, when the pressure was $0.3 \mathrm{MPa}$.
\end{abstract}

Key Words : Bipolar membrane; Phosphorylation; Ferrocene; Separation of monovalent and divalent ions

双极膜 $(\mathrm{BPM})^{[1]}$ 是由阴离子交换膜层、阳离子交 换膜层 ${ }^{[2,3]}$ 复合而成. 因阳、阴膜荷有不同电荷, 使得 双极膜同时具有“篮分效应”与 “荷电效应”, 在一定 的压力差 ${ }^{[4]}$ 下可同时将一价阴、阳离子与高价阴、阳 离子分离[5,6.

二茂铁及其衍生物是一类具有氧化还原中心的 金属有机化合物, 热稳定性好. 它是由两个环戊二烯 基阴离子和一个二价铁离子组成的夹心型化合物,
有芳香性, 是很好的电子传递媒体, 可活化反应物 或提高电子的转移速率. 在盐酸酸性条件下, 二茂 铁可被 $\mathrm{Fe}^{3+}$ 氧化成二茂铁离子[7]: $\mathrm{Fe}^{3+}+\mathrm{Fe}\left(\mathrm{C}_{5} \mathrm{H}_{5}\right)_{2}=\mathrm{Fe}^{2+}+$ $\mathrm{Fe}\left[\left(\mathrm{C}_{5} \mathrm{H}_{5}\right)_{2}\right]^{+}$. 二茂铁离子与海藻酸钠中的羧基发生 螯合反应,生成不溶性的金属有机多聚物.

我国的盐湖卤水中蕴藏有丰富的锂资源, 主要 分布于西藏和青海两省. 从盐湖卤水中提取锂的主 要方法有: 沉淀法、离子交换吸附法、溶剂萃取法、

Received: December 22, 2008; Revised: March 19, 2009; Published on Web: April 16, 2009.

"Corresponding author. Email: zc1224@publ.fz.fj.cn; Tel: +86591-83445289.

福建省自然科学基金(D0710009)资助项目

(C) Editorial office of Acta Physico-Chimica Sinica 
煅烧浸取法、盐析法、碳化法和选择性半透膜法 ${ }^{[8]}$. 壳聚糖季铵盐和海藻酸钠均为天然高分子材 料, 廉价易得, 为环境友好材料, 且具有很好的成膜 性. 本文以甲醛和乙酰基二茂铁为交联剂, 与壳聚糖 交联制备了阴膜溶胶; 以二茂铁离子为交联剂, 与 磷酸化海藻酸钠交联制备了阳膜层. 将阴膜溶胶流 延于阳膜层上制备了 $\mathrm{P}$-二茂铁-SA/乙酰基二茂铁$\mathrm{CS}$ 双极膜(简称为 $\mathrm{P}-\mathrm{mSA} / \mathrm{mCS} \mathrm{BPM}$ ), 该膜具有较高 的电荷密度和离子交换能力. 将 P-mSA/mCS BPM 作为渗透装置的隔膜, 在溶液中离子的初始浓度和 压力差的作用下, 分离盐湖卤水中的一价阴、阳离子 和二价阴、阳离子。

\section{1 实验部分}

\section{1 实验药品及仪器}

海藻酸钠、壳聚糖(脱乙酰度 $90 \%$ )、磷酸三乙 酯、磷酸、五氧化二磷、二茂铁、乙酰基二茂铁、乙酸、 甲醛、 $\mathrm{LiCl} 、 \mathrm{KCl} 、 \mathrm{NaCl} 、 \mathrm{CaCl}_{2} 、 \mathrm{MgCl}_{2}$ 及其他试剂均 为分析纯.

模拟盐湖卤水: 各组分浓度均为 $0.1 \mathrm{~mol} \cdot \mathrm{L}^{-1}$ 的 $\mathrm{LiCl} 、 \mathrm{KCl} 、 \mathrm{NaCl} 、 \mathrm{CaCl}_{2} 、 \mathrm{MgCl}_{2}$ 的混合溶液.

自制的带隔膜的两室电解槽; DF1720SB50型直 流稳压电源(宁波中策电子有限公司); AVATAR360 型红外光谱仪(美国尼高力公司); 北京普析TAS-990 型原子吸收分光光度计(北京威格拉斯有限公司); $\mathrm{Ca} 、 \mathrm{Mg} 、 \mathrm{~K} 、 \mathrm{Na} 、 \mathrm{Li}$ 空心阴极灯(北京威格拉斯有限公 司).

\section{2 双极膜的制备}

取 $3.0 \mathrm{~g}$ 海藻酸钠, 配制成 $3.0 \%$ ( $w$, 质量分数) 海藻酸钠水溶液, 搅拌均匀后, 再减压脱泡, 得到无 色透明的粘稠膜液, 将其流延于平整的玻璃板上, 室

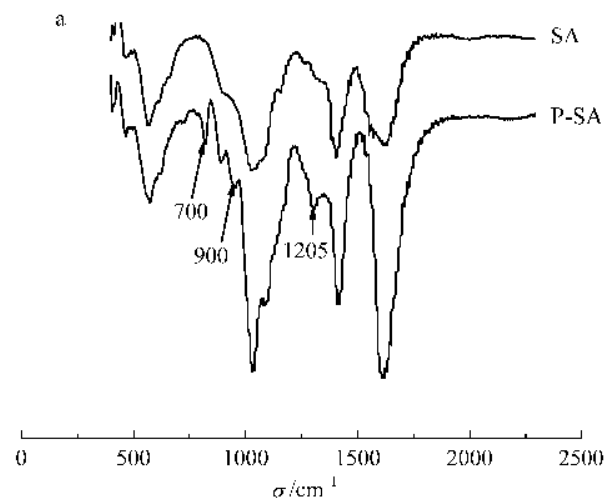

温下风干成膜. 此膜经磷酸化溶液 ${ }^{[9]}(25 \mathrm{~g}$ 五氧化二 磷+42 mL 磷酸三乙酯 $+38 \mathrm{~mL}$ 磷酸)浸泡 $10 \mathrm{~min}$ 后 用蒸馏水淋洗, 去除表面多余的磷酸化溶液, 自然晾 干后在二茂铁离子[7]溶液 $\left(10 \mathrm{~mL} 0.5 \mathrm{~mol} \cdot \mathrm{L}^{-1} \mathrm{FeCl}_{3}\right.$ 溶液 $+1.42 \mathrm{~g}$ 二茂铁 $+10 \mathrm{~mL}$ 盐酸 $+10 \mathrm{~mL}$ 乙醇+15 $\mathrm{mL}$ 水)中浸泡 $15 \mathrm{~min}$, 洗净晾干, 得到蓝色透明薄膜, 即为阳膜层.

取 $3.0 \mathrm{~g}$ 壳聚糖, 用 $2.0 \%(w)$ 的乙酸水溶液摚拌 溶解, 配制成 $3.0 \%(w)$ 壳聚糖乙酸水溶液, 加人 1.0 $\mathrm{mL} 1 \%$ 甲醛和 $0.1 \mathrm{~g}$ 乙酰基二茂铁, 加速搅拌后, 再 减压脱泡, 得到棕红色粘稠的阴膜溶胶, 流延于上述 蓝色阳膜层上, 室温下风干, 即为 $\mathrm{P}-\mathrm{mSA} / \mathrm{mCS}$ 双极 膜.

\section{3 膜的表征及性能测试}

用红外光谱仪分别对改性前后的 SA 和 CS 膜 进行红外测定. 以胶体滴定法 ${ }^{[10,11]}$ 测定 SA 和 CS 的 电荷密度(ED). 以电化学工作站(CHI660C, 上海辰 华仪器有限公司)测定不同频率下双极膜的交流阻 抗频谱图 ${ }^{[12]}$.

\section{2 结果与讨论}

\section{1 双极膜的红外光谱分析}

海藻酸钠磷酸化后, 因引人磷酸根基团, 海藻酸 钠中的氢键作用力减小, 致使 P-SA 红外光谱图中 $800 \mathrm{~cm}^{-1}$ 的羟基峰( $-\mathrm{OH}$ )明显减弱(图 1a). P-SA 在 700-900 和 $1205 \mathrm{~cm}^{-1}$ 处增加新峰, 为磷酸根的伸缩 振动峰 ${ }^{[13]}$, 表明磷酸根与海藻酸钠间发生了化学反 应, SA 成功地接枝上了磷酸根. 磷酸化后, 其表面的 亲水性能得到了明显提高. 如图 2 所示, 磷酸化前 $\mathrm{SA}$ 接触角为 $53.13^{\circ}$, 磷酸化后接触角减小为 $21.67^{\circ}$.

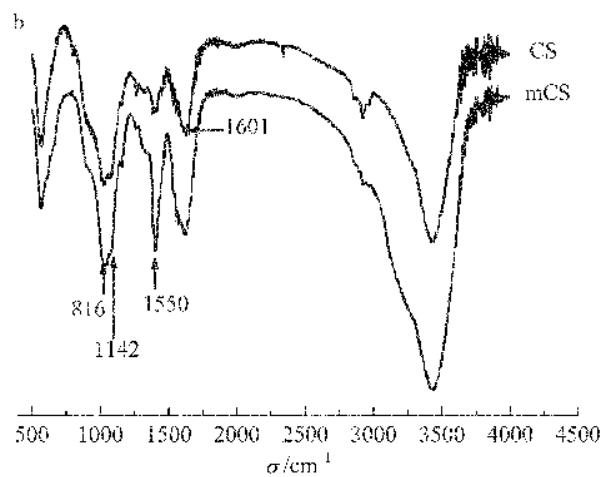

图 1 SA、P-SA、CS 和 $\mathrm{mCS}$ 的红外光谱谱图

Fig.1 Infrared spectra of sodium alginate (SA), phosphorylation sodium alginate (P-SA), chitosan (CS) and modified chitosan (mCS) 


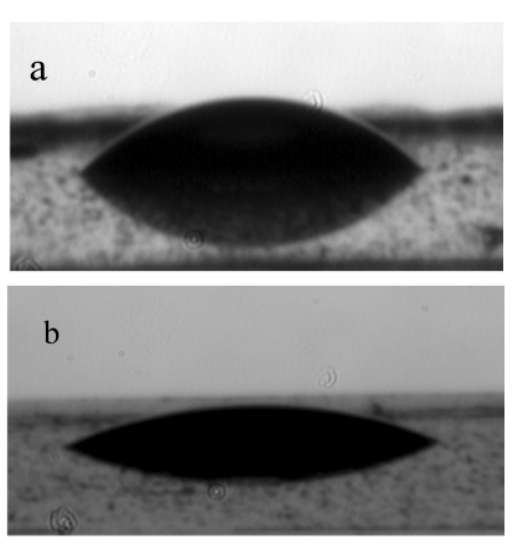

图 2 SA (a) 和 P-SA (b) 的接触角

Fig.2 Contact angles of SA (a) and P-SA (b)

如图 $1 \mathrm{~b}$ 所示, 未经交联的 CS 膜在 $1601 \mathrm{~cm}^{-1}$ 处的吸收峰应归属于壳聚糖的一级氨基特征吸收. 经甲醛和乙酰基二茂铁交联处理后的 $\mathrm{mCS}$ 膜在 $3053 、 1471 、 1142$ 和 $816 \mathrm{~cm}^{-1}$ 附近都出现了二茂铁 基的 $\nu_{\mathrm{C}-\mathrm{H}} 、 \nu_{\mathrm{C}-\mathrm{C} 、} \delta_{\mathrm{C}-\mathrm{H}}$ 和 $\pi_{\mathrm{C}-\mathrm{H}}$ 特征吸收峰 ${ }^{[14]}$; 氨基的 吸收峰红移, 在 $1550 \mathrm{~cm}^{-1}$ 处出现了乙酰基二茂铁与 $\mathrm{CS}$ 中的自由氨基 $\left(-\mathrm{NH}_{3}^{+}\right.$)反应后形成的二级氨基 $\left(-\mathrm{NRH}_{2}^{+}\right)$吸收峰. 交联改性后 $\mathrm{mCS}$ 膜的机械性能 得到了提高 ${ }^{[15]}$.

磷酸化 SA 与二茂铁交联改性过程如图 3 所示. $\mathrm{SA}$ 经磷酸化后, $-\mathrm{PO}_{4}^{3-}$ 接连在了一 $\mathrm{OH}$ 上, 后经二茂
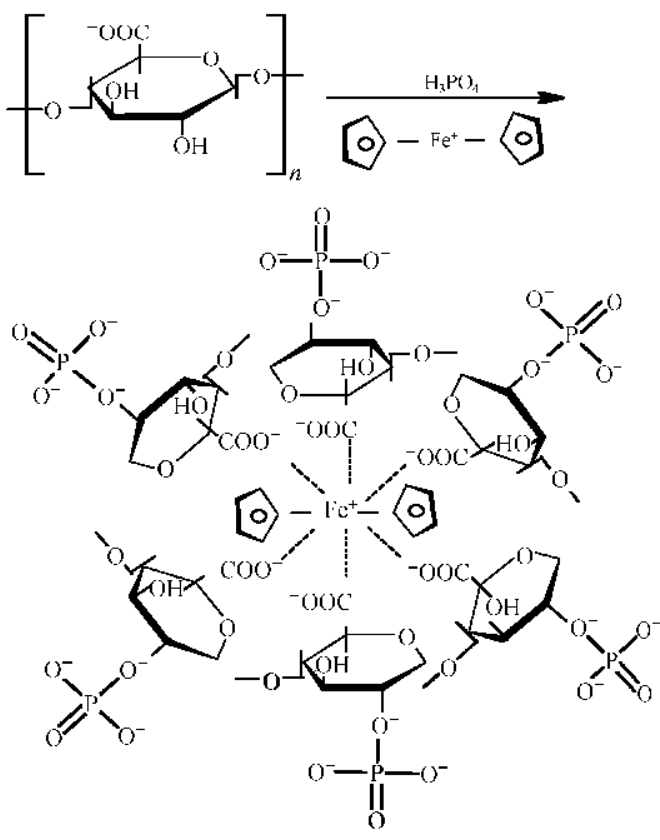

图 3 二茂铁交联改性磷酸化海藻酸钠

Fig.3 Crosslinking process of P-SA modified by ferrocene

铁交联改性, 二茂铁中的 $\mathrm{Fe}^{+}$取代 $\mathrm{Na}^{+}$与一 $\mathrm{COO}^{-}$基 团发生了类似鳌合交联的作用, 从而形成了致密的 网状结构.

壳聚糖溶胶与甲醛和乙酰基二茂铁的交联改性 过程如图 4 所示.
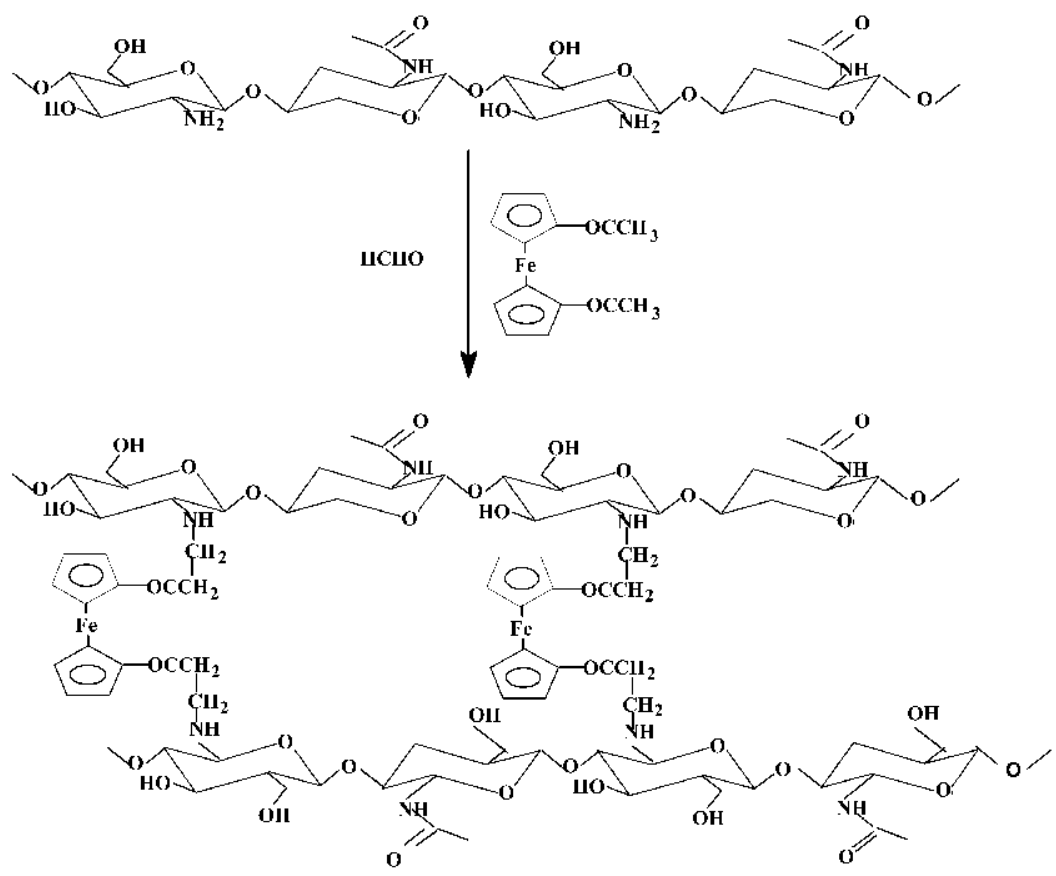

图 4 乙酰基二茂铁交联改性壳聚糖

Fig.4 Crosslinking process of CS modified by acetylferrocene 


\section{2 双极膜的交流阻抗}

双极膜中间层的厚度为纳米级, 可将其视作为 一电容器, $C_{\mathrm{d}}$ 为中间层的容抗, $R_{\mathrm{r}}$ 为其阻抗, 二者并 联后与本体溶液阻抗 $R_{1}$ 相串联, 其等效电路如图 5 中的插图所示. 测试了不同频率 $\left(10^{-1}-10^{5} \mathrm{~Hz}\right)$ 下体系 的阻抗. 以不同频率的 $Z^{\prime}$ 为横坐标, 即实部(体系的 欧姆电阻); 以 $Z^{\prime \prime}$ 为纵坐标, 即虚部(体系的容抗), 高 频半圆和实轴的交点即为膜阻抗, 测得体系的交流 阻抗谱图如图 5 所示. 经磷酸化改性后双极膜的高 频半圆与实轴交点比未经磷酸化改性的双极膜(曲 线 c) 小, 说明磷酸化后膜电阻降低, 导电性能提高. 经二茂铁离子改性的磷酸化双极膜 (曲线 a) 比 $\mathrm{Fe}^{3+}$ 改性的磷酸化双极膜(曲线 b) 具有更小的膜阻抗 ${ }^{[16}$.

\section{$2.3 \mathrm{P}-\mathrm{mSA} / \mathrm{mCS}$ 双极膜在一价、二价离子分离中} 的应用

\subsubsection{P-mSA/mCS BPM 中一价、二价离子的迁移机制}

双极膜的截留机理图如图 6 所示, 阳离子受到 正电层的 Donnan 排斥力被截留; 阴离子受到负电 层的排斥力被截留. 因二价离子比一价离子受到更 强的 Donnan 排斥力, 故双极膜对一价、二价离子具 有选择透过性.

两电解槽之间用 P-mSA/mCS BPM 隔开, 左室 为 $0.10 \mathrm{~mol} \cdot \mathrm{L}^{-1}$ 的 $\mathrm{KCl} 、 \mathrm{NaCl} 、 \mathrm{LiCl} 、 \mathrm{MgCl}_{2}$ 和 $\mathrm{CaCl}_{2}$ 的混合溶液, 右室为 $0.01 \mathrm{~mol} \cdot \mathrm{L}^{-1}$ 盐酸溶液. 膜两侧 溶液体积均为 $100 \mathrm{~mL}$, 膜面积为 $10 \mathrm{~cm}^{2}$, 渗透时间 均为 $3 \mathrm{~h}$. 渗透到右室的阳离子浓度用原子吸收法测 定. 所有实验均在 $298 \mathrm{~K}$ 下进行. 左室通氮气加压.

离子的截留率 $R_{\mathrm{k}}{ }^{[17]}$ 可用式(1)表示:

$R_{\mathrm{k}}=\left(1-\frac{C_{\mathrm{p}, \mathrm{k}}}{C_{\mathrm{m}, \mathrm{k}}}\right) \times 100 \%$

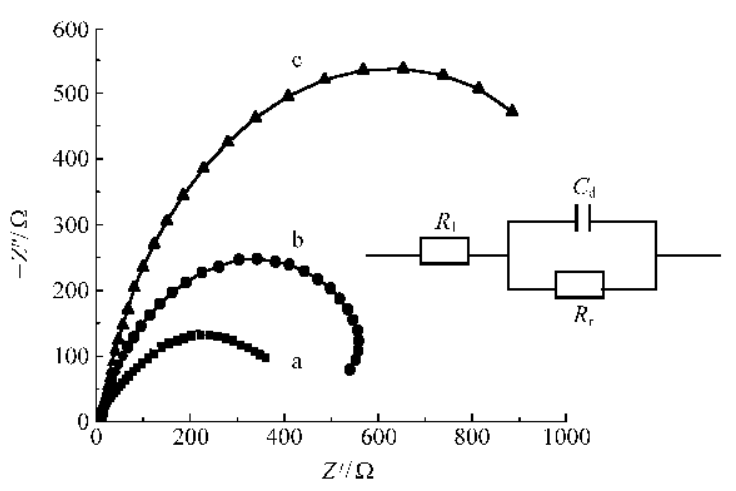

图 5 双极膜(BPM)的交流阻抗频谱图

Fig.5 AC impedances of bipolar membrane (BPM) (a) P-mSA/mCS, (b) P-SA-Fe ${ }^{3+} / \mathrm{mCS}$, (c) $\mathrm{SA} / \mathrm{mCS} ; R_{1}$ : impedance of bulk solution, $C_{\mathrm{d}}$ : internal resistance, $R_{\mathrm{r}}$ : impedance of capacitance; Insert is equivalent electrical circuit.

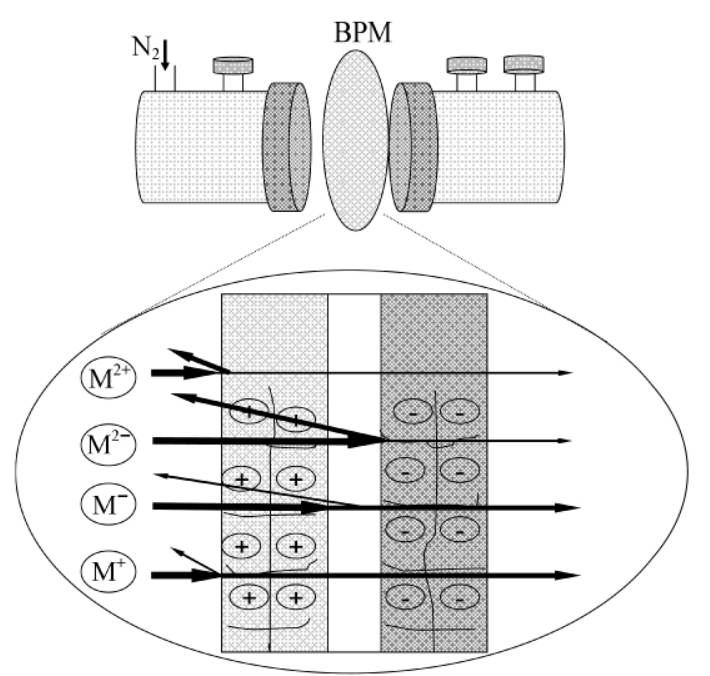

图 6 双极膜的截留模型

Fig.6 Rejection model of bipolar membrane

式中, $C_{\mathrm{p}, \mathrm{k}}$ 为渗透过的金属离子浓度; $C_{\mathrm{m}, \mathrm{k}}$ 为原溶液 的金属离子浓度.

Tsuru 等 ${ }^{\left[{ }^{[17}\right]}$ 用扩展的 Nernst-Planck 方程描述了 浓度、电荷密度及篮分通量三部分对双极纳滤膜中 一价、二价离子迁移的贡献.

\subsection{2一价、二价离子在P-mSA/mCS 双极膜中迁移行为}

(1) 浓度对离子迁移的影响

原始溶液浓度的大小直接影响到一价、二价离 子通过双极膜的迁移量 ${ }^{[17]}$. 如图 7 所示, 当各离子的 初始浓度由 $0.01 \mathrm{~mol} \cdot \mathrm{L}^{-1}$ 增大到 $0.10 \mathrm{~mol} \cdot \mathrm{L}^{-1}$ 时, 因 左、右两室浓度差的增大(右室各离子的初始浓度为 零), 迁移速率随之增大, 各离子的截留率均呈上升 趋势, 与文献[18]相一致.

(2) 双极膜中阴、阳离子电荷密度对截留率的影响

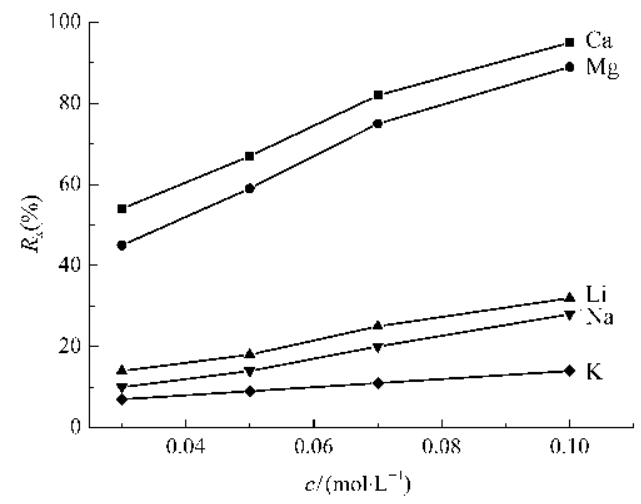

图 7 截留率与渗透室离子浓度的关系

Fig.7 Rejection ratio $\left(R_{\mathrm{k}}\right) v s$ concentration of ions in osmos room $\Delta p=0.0 \mathrm{MPa} ; T=298 \mathrm{~K} ; t=3 \mathrm{~h}$ 
双极膜中阴、阳离子电荷密度的大小也将影响 到一价、二价离子通过双极膜的迁移量 ${ }^{[17]}$. 本研究以 CS、SA 作为阴、阳两膜的基材, 测得 $\mathrm{pH}=4$ 时 CS 中- $\mathrm{NRH}_{2}^{+}$约为 $5.57 \mathrm{mmol} \cdot \mathrm{g}^{-1} ; \mathrm{pH}=10$ 时测得 $\mathrm{SA}$ 中一 $\mathrm{COO}^{-}$的电荷密度约为 $3.78 \mathrm{mmol} \cdot \mathrm{g}^{-1}$. 与所查文 献中的电荷密度值相比(如羧甲基壳聚糖的电荷密 度 $1.18 \mathrm{mmol} \cdot \mathrm{g}^{-1}{ }^{[19]}$, 两种季铵型阳离子淀粉的电荷 密度分别为 0.184 和 $\left.0.215 \mathrm{mmol} \cdot \mathrm{g}^{-1[20]}\right), \mathrm{CS} 、 \mathrm{SA}$ 胶 体具有较高的电荷密度. 实验测得 P-mSA/mCS 双 极膜阳离子、阴离子的交换容量分别为 4.82 和 2.02 $\mathrm{meq} \cdot \mathrm{g}^{-1}$, 表明其具有较高的离子交换能力. 膜中荷 电离子的Donnan 平衡 ${ }^{[2]}$ 如式(2)所示:

$$
\exp \left(\frac{F}{R T}\left(\psi_{l+1}-\psi_{1}\right)\right)=\left(\frac{\gamma_{l, k} C_{l, k}}{\gamma_{l+1, k}} C_{l+1, k}\right)^{\frac{1}{Z_{k}}}
$$

$F$ 为法拉第常数, $R$ 为气体常数 $\left(8.314 \mathrm{~J} \cdot \mathrm{K}^{-1} \cdot \mathrm{moL}^{-1}\right)$, $T$ 为温度 $(\mathrm{K}), C_{k}$ 为离子浓度, $a_{\mathrm{k}}$ 为离子活度, $\gamma$ 为活 度系数, $Z_{\mathrm{k}}$ 为离子化合价, $\psi$ 为电位.

实验测得的一价阳离子的迁移速率远远高于二 价阳离子(图 7). 根据 Donnan 平衡模型 ${ }^{[2]]}$, 形成的 Donnan 位差阻止了同离子从主体溶液向膜内扩散. $\mathrm{P}-\mathrm{mSA} 、 \mathrm{mCS}$ 膜上的活性基团发生解离作用, 在膜 上留下了带荷电的固定基团. P-mSA/mCS 双极膜因 为有较大的离子电荷密度, Donnan 位差也相应较 大, 因此通过P-mSA/mCS 双极膜的一价阴、阳离子 远远高于二价阴、阳离子. 阳离子迁移速率的大小顺 序是 $\mathrm{K}^{+}>\mathrm{Na}^{+}>\mathrm{Li}^{+}>>\mathrm{Mg}^{2+}>\mathrm{Ca}^{2+}$.

(3) 压力差对截留率的影响

左室中各阳离子的浓度均为 $0.1 \mathrm{~mol} \cdot \mathrm{L}^{-1}$, 压力 差从 $0.1 \mathrm{MPa}$ 变化到 $0.4 \mathrm{MPa}$, 截留率随压力差的变 化情况如图 8 所示. 由图 8 可知, 随着压力差的增

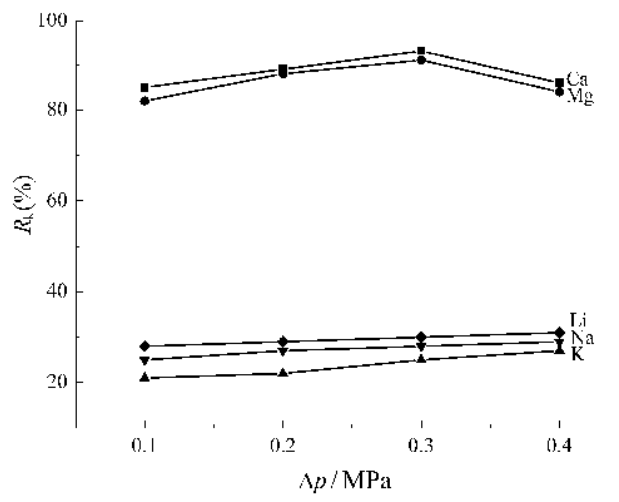

图 8 截留率与压力差的关系

Fig.8 Dependence of rejection ratio on the pressure difference

concentration of metal ions: $0.1 \mathrm{~mol} \cdot \mathrm{L}^{-1} ; T=298 \mathrm{~K} ; t=3 \mathrm{~h}$

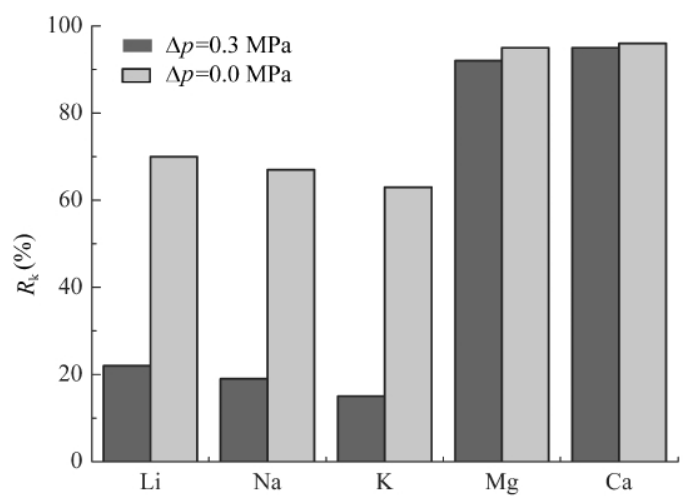

图 9 盐湖卤水中各金属离子透过 P-mSA/mCS BPM 的 截留率

Fig.9 Rejection of metal ions in saline bittern water through P-mSA/mCS bipolar membrane

concentration of metal ions : $0.1 \mathrm{~mol} \cdot \mathrm{L}^{-1} ; T=298 \mathrm{~K} ; t=3 \mathrm{~h}$

大, $\mathrm{Ca}^{2+} 、 \mathrm{Mg}^{2+}$ 的截留率先增大后减小; 一价离子的 截留率逐渐增大, 最后达到一恒定值. 当压力差达到 $0.3 \mathrm{MPa}$ 时, 二价离子的截留率最大; 一价离子的截 留率基本恒定. 当压力差超过 $0.3 \mathrm{MPa}$ 时, 二价离子 的截留率减小. 这是因为当压力差大到一定程度, 双 极膜上所带的电荷还来不及和二价离子发生选择交 换作用就被渗透进人右室中, 从而影响了一价离子 和二价离子的分离.

\subsection{3 盐湖卤水中一价、二价阳离子的分离}

模拟盐湖卤水 ${ }^{[22]}$ 中的各阳离子通过P-mSA/mCS 双极膜的结果如图 9 所示. 运行 $3 \mathrm{~h}$ 后, 当压力差为 $0.3 \mathrm{MPa}$ 时, $\mathrm{K}^{+} 、 \mathrm{Na}^{+} 、 \mathrm{Li}^{+}$的截留率明显低于 $\mathrm{Mg}^{2+}$ 和 $\mathrm{Ca}^{2+}$. 一价离子的截留率小于 $22 \%$, 明显地低于无压 力差下的截留率; 压力差对二价离子截留率的影响 不大.

\section{3 结 论}

以磷酸化海藻酸钠与二茂铁离子交联制备了阳 离子交换膜层, 以甲醛和乙酰基二茂铁改性壳聚糖 制备了阴离子交换膜层, 用流延法制备了 P-mSA/ $\mathrm{mCS}$ 双极膜. 红外分析结果表明, 阴膜层与乙酰基 二茂铁、甲醛发生了 Mannish 反应, 阳膜层交联上了 磷酸根基团和二茂铁离子. 改性后的双极膜具有更 好的亲水性和机械性能, 双极膜的阻抗明显减小. 用 改性后的 P-mSA/mCS 双极膜处理盐湖卤水, 当压 力差为 $0.3 \mathrm{MPa}$ 时,一价阳离子的截留率小于 $22 \%$, 二价阳离子的截留率高于 $95 \%$, 压力差对二价离子 截留率的影响不大. 


\section{References}

1 Mafe, S.; Ramirez, P.; Alcaraz, A.; Aguilella, V. M. Handbook of bipolar membrane technology. Enschede: Twente University Press, 2000: 49-78

2 Hsueh, C. L.; Peng, Y. J.; Wang,C. C.; Chen, C. Y. J. Membr. Sci., 2003, 219: 1

3 Xu, T. W.; Fu, R. Q.; Yang, W. H. J. Membr. Sci., 2006, 279: 233

4 Richard, B. W.; Mukhtar, H. J. Membr. Sci., 1996, 112: 263

5 Urairi, U.; Tsuru, T.; Nakao, S.; Kimura, S. J. Membr. Sci., 1992, 70: 153

6 Guo, L. Z.; Shi, P. J. Environmental Science Guide, 2008, 27: 17 [郭岭志, 施培俊. 环境科学导刊, 2008, 27: 17]

7 Jiang, L.; Di, X. W.; He, Z. X. Metallurgical Analysis, 2006, 26: 59 [姜 㝵, 狄晓威, 贺忠翔. 治金分析, 2006, 26: 59]

8 Chen, T.; Kang, Z. H. Guangdong Trace Elements Science, 2007, 14:6 [陈 婷, 康自华. 广东微量元素科学, 2007, 14: 6]

9 Granja, P. L.; Barbosa, M. A. J. Mater. Sci., 2001, 36: 2163

10 Xu, C. X.; Chen, R. Y.; Zheng, X.; Chen, X.; Chen, Z. J. Appl. Polym. Sci., 2008, 107: 3076

11 Huang, Z. X.; Chen, R. Y.; Zheng, X.; Chen, X.; Chen, Z. Acta Phys. -Chim. Sin., 2007, 23: 1771 [黄振霞, 陈日耀, 郑 䂀, 陈 晓, 陈 震. 物理化学学报, 2007, 23: 1771]

12 Hurwitz, H. D.; Dibiani, R. J. Membr. Sci., 2004, 228: 17
13 Zheng, G. W.; Gong, C. L.; Wen, S.; Xie, X. L. Acta Phys. -Chim. Sin., 2008, 24: 855 [郑根稳, 龚春丽, 文 胜, 解孝林. 物理化 学学报, 2008, 24: 855]

14 Gao, S. P.; Zhang, J. X.; Li, B. J. North China Institute of Technology, 2004, 25: 281 [高松平, 张俊祥, 李 冰. 华北工学 院学报, 2004, 25: 281]

15 Xu, C. X.; Chen, R. Y.; Zheng, X.; Chen, X.; Chen, Z. J. Membr. Sci., 2008, 307: 218

16 Wang, Y. H.; Ma, X. L.; Chen, R. Y.; Zheng, X.; Chen, Z. Acta Phys. -Chim. Sin., 2008, 24: 1041 [王嫣红, 马秀玲, 陈日耀, 郑 曦, 陈 震. 物理化学学报, 2008, 24: 1041]

17 Tsuru, T.; Nakao, S.; Kimura, S. J. Membr. Sci., 1995, 108: 269-278

18 Higuchi, A.; Nakagawa, T. J. Membr. Sci., 1987, 32: 267

19 Chen, H. F.; Pan, S. R.; Hu, Y.; Zhong, Y. S. J. Instrumental Analysis, 2003, 22: 70 [陈浩凡, 潘仕荣, 胡 瑜, 钟跃思. 仪器分析, 2003, 22: 70]

20 Ren, Y. X.; Chen, L. Q.; Chen, R. Y.; Zheng, X.; Chen, Z. J. Membr. Sci., 2007, 27: 72

21 Higuchi, A.; Nakagawa, T. J. Membr. Sci., 1987, 32: 267

22 Xu, T. W.; Yang, W. H.; Sun, S. S.; Liu, Z. M.; Li, S. Q.; Li, X. D. Technology Progress, 2001, 6: 20 [徐铜文, 杨伟华, 孙树声, 刘兆明, 李善清, 李旭娣. 技术进展, 2001, 6: 20] 